\title{
Research on Energy-Saving Charging Combination Based on Group Genetic Algorithm (GGA) and Best Fit (BF) Algorithm
}

\author{
Zhu Baiqing ${ }^{1}$, Lu Haixing ${ }^{2}$, Tong Yifei ${ }^{2}$, Li Dongbo $^{2, *}$ and Xia Yong ${ }^{1}$ \\ ${ }^{I}$ School of economics and management, Nanjing Institute of Technology, Nanjing, 211167, People's Republic of China; \\ ${ }^{2}$ School of Mechanical Engineering, Nanjing University of Science and Technology, Nanjing 210094, People's Republic \\ of China
}

\begin{abstract}
As a traditional high energy-consuming industry, the forging industry consumes a lot of energy. The activity consuming highest energy during forging process is the forging and heating. In order to solve the problem regarding how to separate work pieces with different holding temperature intervals and combine them for charging, a group of work pieces with compatible holding temperatures are analyzed for energy-saving charging combination. To aim this, an optimal charging combination model for $t$ energy saving is proposed as well as a group genetic algorithm based on temperature compatibility rule for problem solving. To obtain better optimal solutions, a BF (best fit) heuristic algorithm is designed to generate individuals and initial population as well as an improved crossover operator. At last an instance is analyzed to verify the effectiveness of the proposed model and algorithm, and compared with the present charging planning used in forging enterprises.
\end{abstract}

Keywords: BF (best fit), charging combination, energy saving, group genetic algorithm, temperature interval.

\section{INTRODUCTION}

As a traditional high energy-consuming industry, the forging industry consumes a lot of energy every year, occupying approximately $20 \%$ of total cost in a factory. In order to save energy and therefore increase the profit, more and more enterprises consider the performance per watt as a key factor to win competition [1]. The procedure consuming highest energy during forging process is the forging [2].

For forging production, production planning is to separate, combine and arrange the work pieces to be charged and forged [3], called as job scheduling [4]. A lot of researches focusing on flow shop scheduling or job shop scheduling have been carried out with minimum time of completion [5] (i.e. typical minimum Makespan problem) or minimum delivery delay as optimization objective. Another kind of batch scheduling of interval production also aims to minimum the completion time [6]. However, blanks sent to a forging shop in actual productions are usually made of different kinds of materials, which have different holding temperatures for heating before forging. When blanks are separated into batches for charging, it is scarcely possible to ensure all blanks in a batch are made of the same material. Otherwise a batch would comprise only several pieces of blanks, far less than optimal charging amounts, resulting in decreased equipment performance due to the sharply increased heating energies as well as delay of lead time. So it is impractical to separate blanks only by materials.
Charging combination is less studied and energy saving is seldom considered for forging production optimization [7]. In this paper, the charging combination for forging production is studied and an optimization model of energy-saving charging combination is established with considerations of temperature interval. When making process specifications, an interval temperature instead of a temperature value is usually presented according to the products performance requirements by customers, because of the heating of blanks in actual production strictly specified, limited processing technology of business and field measurement error. For example, the holding temperature of work piece i is $\left[T_{i_{\min }}, T_{i_{\max }}\right\rfloor$, where $T_{i_{\min }}$ refers to the holding temperature the work piece shall reach and $T_{i_{\max }}$ refers to the holding temperature the work piece shall not exceed. The product performances will not be affected, as long as the holding temperature for blanks falls into this defined interval. So blanks made of different kinds of materials with different holding temperatures can be arranged in a batch and heated in a furnace together, provided their holding temperatures have an intersection. Therefore, it is possible in this case to optimize blanks charging for energy saving.

\section{PROBLEM DESCRIPTION}

Both the weights of a charging batch and the holding temperature (e.g. whether holding temperatures of blanks in a charging batch have an intersection) shall be considered for batch production. In order to optimize the charging combination for energy saving, batching should comprehensively aims to minimize the quantity of charging batches, maximize 
the average charging amount and keep the average holding temperature of all batches as low as possible.

For convenient description of the charging problem, the average holding temperature of a batch and the temperature compatibility are defined as follows:

Definition 1: Average holding temperature of a batch (called as AHT hereinafter for short) refers to the average value of final holding temperatures of all batches in a batching plan. That is,

$T=\frac{1}{k} \sum_{b=1}^{k} T_{b}$

where $\mathrm{T}$ denotes the average charging temperature, denotes the quantity of charging batches and denotes the holding temperature of $b_{\text {th }}$ batch $(b=1,2, \ldots, k)$.

Definition 2: Temperature compatibility: the holding temperature intervals of work pieces $i$ and $j$ are $\left[T_{i \min }, T_{i \max }\right]$ and $\left[T_{j \min }, T_{j \max }\right\rfloor$ respectively and if Equation (2) is satisfied, work piece $\mathrm{i}$ and $\mathrm{j}$ are called temperature compatible, which means they can be heated in a same furnace. If not, they cannot be batched for heating in a same furnace.

$\left[T_{i \min }, T_{i \max }\right] \cap\left\lfloor T_{j \min }, T_{j \max }\right\rfloor \neq \phi$

This optimization issue regarding charging combination for energy-saving forging, discussed here, can be described as follows.

There are $\mathrm{N}$ work pieces (the weight and the holding temperature interval of each work piece have been given) ready to be separated into $\mathrm{B}$ batches for heating. Each batch refers to a heating job in a furnace and the maximum charging capacity of furnace is $\mathrm{S}$. The work pieces in a batch should be compatible and the total weights shall not exceed $\mathrm{S}$. The optimization objective is to minimize the quantity of charging batches, maximize the average charging amount and keep the average holding temperature of all batches as low as possible.

\section{ENERGY-SAVING CHARGING COMBINATION BASED ON TEMPERATURE INTERVAL}

\subsection{Basic Premise}

(1) Each work piece is allocated to a charging batch.

(2) A work piece would be subject to several heating steps. For example, it would be heated again after forging. Because its initial temperature, finishing temperature and heating time are all defined in process specifications and its tapping temperature is always the same, it can be considered as a constant heating process with the heating steps being ignored. It is assumed that each batch is only heated one time in one furnace.

(3) A batch of work pieces are charged and heated at the same time. Once the heating process is started, interruption is not allowed, which means any work piece cannot be taken out of the furnace before reaching the final temperature. In addition, new work piece shall not be added into the furnace before current heating is finished.

(4) The capacity of the processing next to forging (machining) is sufficient.

(5) The scheduled job does not exceed the maximum capacity of equipments.

(6) The biggest weight of work piece does not exceed the maximum loading capacity of heating furnace.

(7) Work pieces in a batch have similar holding time, so the constraint of minimum total processing time can be ignored.

(8) The furnace is equipped with sufficient heating capacity and the temperature the furnace can reach covers holding temperatures of all work pieces.

\subsection{Notations}

The notations used in this paper are as follows:

$\mathrm{n}$ : quantity of work pieces;

$\mathrm{k}$ : quantity of batches;

$\mathrm{J}$ : work pieces set, $J=\{1,2, \ldots, \mathrm{n}\}$;

B: charging batches set, $B=\{1,2, \ldots, k\}$;

$\mathrm{j}$ : serial number of a work piece, $j \in J$;

$\mathrm{b}$ : serial number of a charging batch, $\mathrm{b} \in B$;

$J_{b}$ : work pieces set in batch $\mathrm{b}, \mathrm{b} \in B \quad J_{b} \in J$;

$z_{j}:$ weight of work piece $\mathrm{j}$;

$z_{b}$ : weight of charging batch $\mathrm{b}$;

$\mathrm{S}$ : maximum load capacity of furnace;

$T_{j_{\max }}$ : upper limit of holding temperature for work piece $\mathrm{j}$

$T_{j_{\min }}$ : lower limit of holding temperature for work piece $\mathrm{j}$;

$T_{b}$ : holding temperature of charging batch $\mathrm{b}$ in furnace;

$K_{L B}$ : lower quantity limit of charging batches;

$x_{j b}$ : decision variable to determine whether work piece $\mathrm{j}$ to heat in batch $b$ );

$Q$ : queue for work pieces;

$Q^{\prime}$ : queue for charging batches.

\subsection{Mathematical Model}

The following mathematical model can be established according to the above premise and definitions: 


$$
\begin{aligned}
& \min (k) \\
& \min Q=\frac{1}{k} \sum_{b=1}^{k 1}\left(\begin{array}{ll}
S \quad Z_{b}
\end{array}\right) \\
& \min T=\frac{1}{k} \sum_{b \in B} T_{b} \\
& \sum_{b \in B} \mathrm{x}_{j b}=1, j \in J \\
& z_{b}=\sum_{j \in J} x_{j b} z_{j} \leqslant S, b \in B \\
& \left.\bigcap_{j \in J_{b}} T_{j \min } T_{j \max }\right\rfloor \neq \phi, \quad b \in B \\
& \left.T_{b}=\max _{j_{\min }}\right\}_{j \in J_{b}, b \in B} \\
& \sum_{j=1}^{n} z_{j} / S \leq k \leq n \\
& x_{j b}=\sum_{1, j \in J_{b}} 0, j \notin J_{b} \\
& Q_{\text {total }}=\left[Q_{\text {service }}, Q_{\text {VO }}, Q_{\text {resource }}\right]
\end{aligned}
$$

Equations (3), (4) and (5) are optimal objective functions with minimum quantity of charging batches, minimum average charging difference and minimum average holding temperature respectively. Equation (6) indicates each work piece $\mathrm{j}$ can be allocated to only one charging batch $\mathrm{b}$. Equation (7) is the constraint for batch weight, indicating the total weight of work pieces in a batch shall not exceed the maximum load capacity of the furnace. Equation (8) indicates the intersection of holding temperature intervals of all work pieces in a batch and shall be not void, which means all work pieces in a charging batch shall be temperature compatible. Equation (9) indicates the holding temperature for a charging batch and shall be defined in a manner all work pieces can be covered but as low as possible. Equation (10) defines the quantity range of charging batches by a lower limit, i.e. $\sum_{j=1}^{n} z_{j}$. It is assumed that work pieces can be separated and allocated to different batches. In addition, all work pieces in a batch shall be temperature compatible so that a limit value is generated. Equation (11) is the decision variable.

\section{GROUP GENETIC ALGORITHM SOLUTION BASED ON TEMPERATURE COMPATIBILITY RULE}

The group genetic algorithm (GGA)[8] is adopted in this paper to solute the optimizations. GGA is different than other genetic algorithms in its coding way and genetic operations [9]. In the practical utilization of GGA, with several heuristic algorithms considered into GA operations (e.g. population initialization and crossover) based on model features, a group genetic algorithm based on temperature compatibility rule is proposed.

\subsection{Population Initialization Based On BF (Best Fit) Heuristic Algorithm}

For an intelligent algorithm, normally the first step is to establish mapping relationship between individuals and problem domain. The coding defined in the group genetic algorithm is used to code individuals, which are then represented in the form of a two-dimensional array. This coding can effectively reduce the redundant information.

Before coding, work pieces shall be order and numbered. A chromosome in the code corresponds to a batching plan and the length of chromosome to the quantity of work pieces. The value of genes, which are grouped, denotes the serial number of work piece in group. An example is as shown in Fig. (1). It indicates that work pieces 5 and 12 are allocated to batch B1, work pieces 2, 7 and 10 to batch B2, work pieces 8 and 11 to batch B3, work pieces 1, 4, 6 and 9 to batch B4, and work piece 3 to batch B5.

$$
\text { X: } \begin{array}{|l|l|l|l|l|}
\hline 512 & 2710 & 811 & 1469 & 3 \\
\hline
\end{array}
$$

Fig. (1). Code illustration.

When generating individuals, work pieces in a charging batch have to be constrained by the batch weight and the temperature compatibility rule, so a BF (best fit) heuristic algorithm based on constraints of batch weight and temperature compatibility [10] is designed to generate individuals and furthermore the initial population as shown in Fig. (2).

Step 1: Work pieces are randomly ordered in queue Q and numbered $(1,2, \ldots, n)$.

Step 2: Get work piece j out of Q. denotes the weight of work piece $\mathrm{j}$ and for its holding temperature interval.

Step 3: $\ominus$ Select batch $b$ which has not been matched with work piece $\mathrm{j}$ from batches queue $\mathrm{Q}^{\prime}$ and check whether work piece $\mathrm{j}$ suits batch $\mathrm{b}$. The weight margin of batch $\mathrm{b}$ is $\Delta s_{b}=S-s_{b}\left(s_{b}\right.$ is the current weight of batch $\left.\mathrm{b}\right)$ and its temperature interval is $T_{b}$ ( $T_{b}$ is the intersection of holding temperature intervals of all work pieces in batch $b$ and shall be not void). $\ominus$ If all batches in Q' have been matched with work piece j, create a new batch $b_{\text {new }}$ in Q' and arrange work piece $\mathrm{j}$ into it. Update the residual weight margin $\Delta s_{b_{\text {new }}}=S-z_{j}$ and the temperature interval $T_{p}=T_{j}$ of batch $b_{\text {new }}$. Until Q contains no work piece unmatched, go to Step4, otherwise Step2. If there is a batch in Q' which has not been matched with work piece $\mathrm{j}$, calculate $\Delta s_{b}{ }^{\prime}=\Delta s_{b}+z_{j} \quad$ and $T_{b}{ }^{\prime}=T_{j} \bigcap T_{b}$. If $\quad \Delta s_{b}{ }^{\prime} \geq 0$, $T_{b}{ }^{\prime} \neq \phi$, arrange work piece $\mathrm{j}$ into batch $\mathrm{b}$ and update the residual weight margin $\Delta s_{b}=\Delta s_{b}^{\prime}$ and the temperature interval $T_{b}=T_{b}^{\prime}$ of batch b. Until Q contains no work 


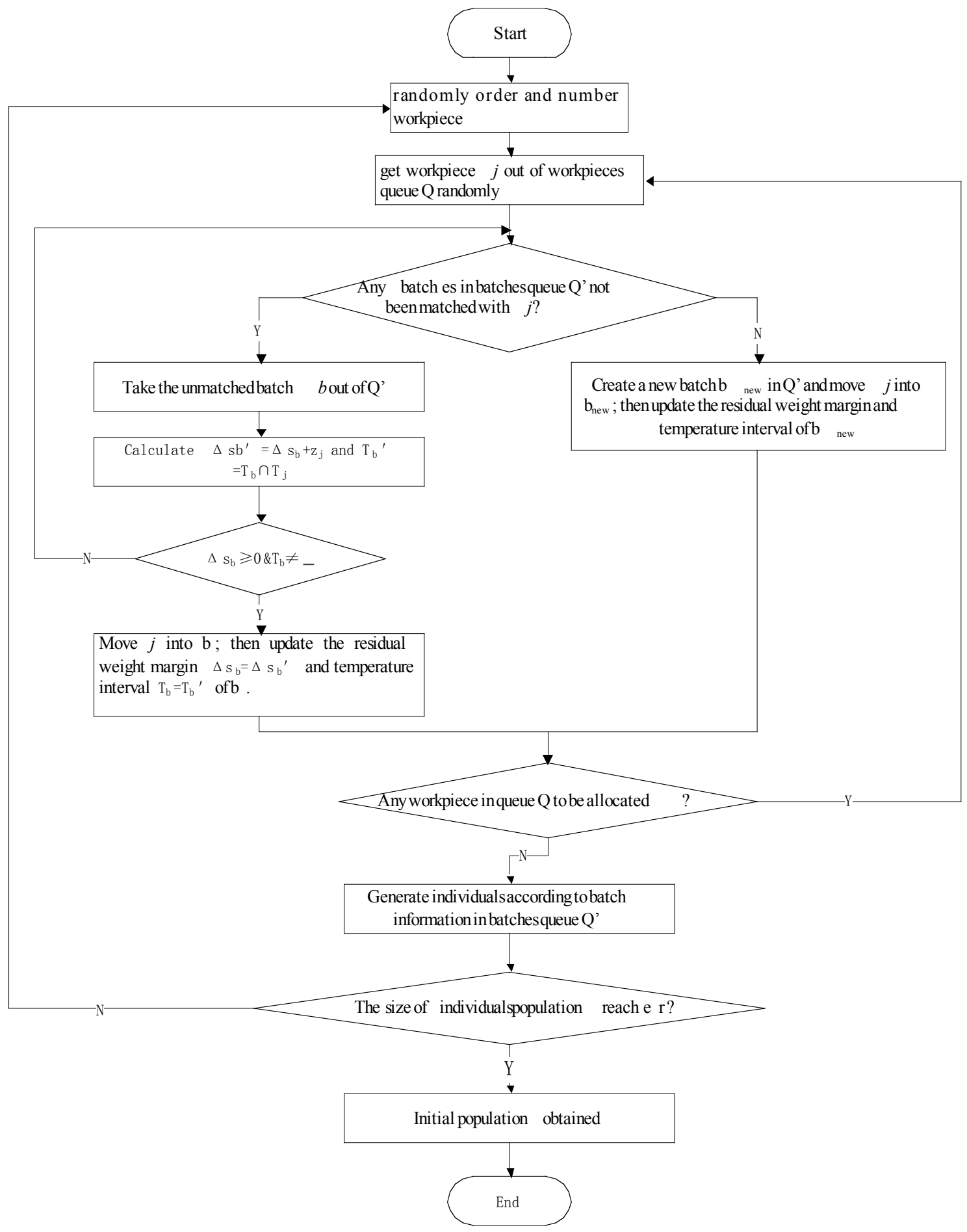

Fig. (2). Population initialization based on BF.

piece unmatched, go to Step4, otherwise Step2. If $\Delta s_{b}{ }^{\prime}<0$ or $T_{b}^{\prime}=\phi$, repeat Step 3 .

Step 4: After each work piece has been allocated to corresponding batch in queue Q', a batching plan is formed. Use the coding way mentioned above to generate the individual. Then go to Step1 to generate a new individual until the predefined population size is reached.

\subsection{Group Genetic Algorithm Based On Temperature Compatibility Rule}

(1) Coding and population initialization

When initializing population, random individual generation may result in infeasible solution. Because a twodimensional array is used to code individuals, the BF heuristic algorithm with constraints of temperature compatibility and batch weight described above is used for population initialization. The population which is initialized by the BF 
algorithm can not only ensure the feasibility of each individual, but also the randomness for the initial population to be uniformly distributed in the solution domain.

\section{(2) Fitness function}

This proposed model comprehensively consider the optimization objectives as minimum quantity of charging batches, minimum average charging difference and minimum average holding temperature. So the comprehensive evaluation with linear weighting is used to determine the fitness function, as expressed below:

$$
\begin{aligned}
& \text { fitness }=w_{k} \frac{k_{\max }-k}{k_{\max }-k_{\min }}+w_{q} \frac{q_{\max }-q}{q_{\max }-q_{\min }}+w_{T} \frac{T_{\max }-T}{T_{\max }-T_{\min }} \\
& q=\frac{1}{k-1} \sum_{b=1}^{k-1}\left(S-Z_{b}\right) \\
& T=\frac{1}{k} \sum_{b \in B} T_{b}
\end{aligned}
$$

where $w_{k}, w_{q}$ and $w_{T}$ represent the weighting coefficients of quantity of charging batches, average charging difference and average holding temperature respectively and $w_{k}+w_{q}+w_{T}=1 . k_{\max }, k_{\min }, q_{\max }, q_{\min }, T_{\max }$ and $T_{\text {min }}$ represent biggest and smallest quantity of charging batches, biggest and smallest average charging difference, biggest and smallest average holding temperature and average holding temperature of each generation.

\section{(3) Selection}

The roulette algorithm is adopted to select individuals [11].

\section{(4) Crossover}

The crossover operation is mainly used to generate filial generation. If the filial generation can retain excellent gene segments from parents, it will be more effective. The crossover is related to coding and bad or improper crossover can negatively affect the algorithm performance. An improved crossover operator is proposed for group genetic algorithm described here.

Step 1: Randomly select $[k / 2]$ charging batch segments from parents $F_{1}$ (where refers to the quantity of charging batches).

Step 2: Select the charging batch segment from parents $F_{2}$ (this batch shall have a work piece which is not included in $S_{1}$ ) and then put it into $S_{1}$.

Step 3: If the length of $S_{1}$ satisfy the length of chromosome code, go to Step8, otherwise Step4.

Step 4: Remove the work piece which is not included in $S_{1}$ from $F_{2}$ and put residual charging batch segments into queue $Q_{1}$.
Step5: Combine those charging batch segments in $Q_{1}$ with consideration of two constraints: temperature compatibility rule and batch weight discussed above. The quantity of work pieces in each combination varies from 1 to $\mathrm{v}(\mathrm{v}$ is the biggest quantity of work pieces of batches in $Q_{1}$ ). Then put them into $Q_{2}$.

Step 6: Combination the work pieces (the number of combined work pieces from 1 to $\mathrm{g}$ ) of each batch in $S_{1}$ (g refers to the quantity of work pieces in each batch) and then put the combinations into $u_{1}, u_{2} \ldots u_{l}$ respectively ( $l$ is the quantity of charging batches in $F_{3}$ ).

Step 7: Use combinations in $Q_{2}$ to recombine with charging combinations in $u_{1}, u_{2} \ldots u_{l}$ one by one with the same constraints in Step5 and then put the recombination into $S_{2}$. It is required that the batch weight shall be as big as possible as long as the constraints are satisfied. Put the exchanged work pieces into $Q_{2}$ and remove the work piece which can be recombined out of $Q_{2}$.

Step 8: For work pieces in $Q_{2}$, which are not included in $S_{2}$, combine them according to BF and then put them into $S_{2}$. So $S_{2}$ is the filial generation generated from parents $F_{1}$ and $F_{2}$ through crossover.

Step 9: Exchange positions of $F_{1}$ and $F_{2}$. Then repeat Step1 to Step8 until another filial generation is obtained.

Below is a crossover example.

There are two parents $F_{1}$ and $F_{2}$ as shown in Fig. (3). Select 2 nd and 4 th batch segments from and put them into. Then select batch segments from which do not include those work pieces in $2^{\text {nd }}$ and $4^{\text {th }}$ batch from $F_{1}$ and put them into $S_{1}$ so as to form $S_{1}$. Then remove work pieces which have been included in $S_{1}$ from $F_{2}$ to form $Q_{1}$. Put work pieces included in $Q_{1}$ into $Q_{2}$ and $Q_{2}:(1)(9)(1,9)(1,15)(3,5,11)$.

$$
\begin{aligned}
& \mathrm{F}_{1}: \begin{array}{|lll|lll|llll|lll|ll|}
\hline 4 & 5 & 11 & 2 & 8 & 10 & 1 & 7 & 13 & 15 & 6 & 12 & 14 & 3 & 9 \\
\hline
\end{array} \\
& F_{2}: \begin{array}{lllllllllll|lll|lll|}
3 & 5 & 11 & 14 & 4 & 7 & 13 & 8 & 15 & 1 & 6 & 12 & 2 & 9 & 10 \\
\hline
\end{array}
\end{aligned}
$$

Fig. (3). Chromosome codes of two parents.

Note that in case only considering the batch weight constraint, segments $(9,15)$ and $(1,9,15)$ will be unnecessarily generated. However, since work pieces 9 and 15 are not temperature compatible, the two infeasible solutions are avoided. 


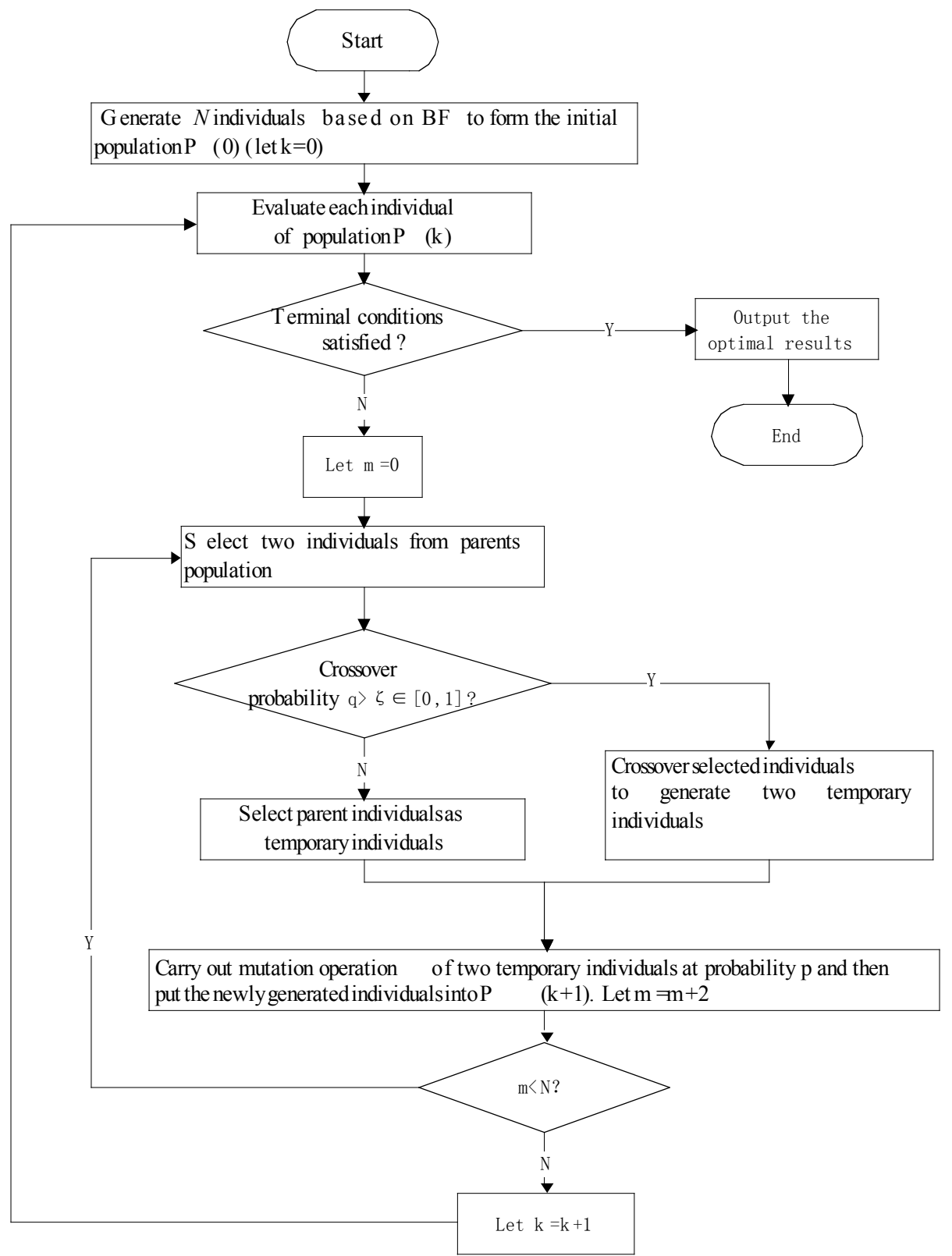

Fig. (4). Group genetic algorithm solution based on temperature compatibility rule.

After carrying out combination on batch segments in $S_{1}$, the following can be obtained:

$$
\left(u_{1}, u_{2}, u_{3}\right)=\left(\begin{array}{ccc}
(2) & (6) & (4) \\
(8) & (12) & (7) \\
(10) & (14) & (13) \\
(2,8) & (6,12) & (4,7) \\
(2,10) & (6,14) & (4,13) \\
(8,10) & (12,14) & (7,13) \\
(2,8,10) & (6,12,14) & (4,7,13)
\end{array}\right)
$$

After combining segments in $u_{1}$ with batch segments in $Q_{2}$, the following can be obtained. It can be seen that 10 is replaced by $(1,9) .(1,9)$ and related segments are removed from $Q_{2}$ and replaced by 11 . Provided the batch segments after recombination are: $(1,2,8,9)(6,10,12,14)(4,7,13)$. Then use BF method to combine so as to form the final individual $S_{2}$ with work pieces which are not included in $Q_{2}$. This recombination make the charging amount of each batch as much as possible with the advantage of this crossover operator to generate batch weight closer to load capacity.

\section{(5) Mutation}

The mutation operation in group genetic algorithm is normally used to delete one or several gene segments for recombination. The mutation operation used in this paper is consisted of the following steps: Randomly select several charging batches. If their average charging rate is below $\eta$ 
Table 1. Parameters of workpieces.

\begin{tabular}{|c|c|c|c|}
\hline $\mathrm{J} 1$ & 4 & 624 & {$[1200,1250]$} \\
\hline $\mathrm{J} 2$ & 3 & 726 & {$[1250,1300]$} \\
\hline $\mathrm{J} 3$ & 12 & 359 & {$[1180,1230]$} \\
\hline $\mathrm{J} 5$ & 11 & 289 & {$[1100,1180]$} \\
\hline $\mathrm{J} 6$ & 3 & 512 & {$[1230,1300]$} \\
\hline $\mathrm{J} 7$ & 2 & 717 & {$[1320,1400]$} \\
\hline $\mathrm{J} 10$ & 16 & 798 & {$[1280,1350]$} \\
\hline $\mathrm{J} 11$ & 1 & 1364 & {$[1390,1450]$} \\
\hline $\mathrm{J} 12$ & 6 & 823 & {$[1250,1320]$} \\
\hline $\mathrm{J} 13$ & 5 & 334 & {$[1130,1180]$} \\
\hline $\mathrm{J} 14$ & 4 & 669 & {$[950,1000]$} \\
\hline $\mathrm{J} 15$ & 17 & 258 & {$[900,980]$} \\
\hline $\mathrm{J} 16$ & 7 & 407 & {$[1000,1080]$} \\
\hline
\end{tabular}

(such as 0.8), take all work pieces out of those batches to cancel the batch and then randomly order. Use BF method to re-separate those work pieces into batches.

The group genetic algorithm solution based on temperature compatibility rule is shown in Fig. (4).

\section{CASE STUDY}

Take an actual multi-order charging planning from DVR (a famous forging enterprise in China) as example to illustrate the solutions of proposed algorithm above. The work pieces forms the same order is classified into the same type with similar holding time. Therefore when work pieces are separated into batches, the constraint of maximum load capacity of heating furnace and the holding temperature compatibility among different types of work pieces shall be taken into consideration. The maximum load capacity of heating furnace is $8 \mathrm{~T}$ and related parameters of work piece are listed in Table 1.

The traditional batching in DVR is usually planned based on the following principles: First separate work pieces into different aggregates and calculate total weight of each aggregate. For work pieces which are holding temperature compatible but weight of which exceeds maximum capacity of furnace, the extra work pieces will be put into a new batch for charging until each batch is not overweight. Then regarding those aggregates as individuals, order them with descending weights and initially consider each aggregate as a charging batch. Start batch matching from the batch with the biggest weight. If two or more aggregates can be combined into a batch, combine them. Otherwise take it as a single batch.

The batching planned by the traditional way is as follows: number of batches is 12 , average charging amount is $5455 \mathrm{~kg}$ and the average holding temperature is $1171^{\circ} \mathrm{C}$. The details of charging batches are shown in Table 2 .

The group genetic algorithm based on temperature compatibility rule described in Section 4 in this paper is used to figure out a optimal batching plan. Parameters involved are listed in Table 3. Repeatable run the algorithm 50 times so as to obtain the following optimal solution: the number of batches is 10 , the average charging amount is $6587 \mathrm{~kg}$ and the average holding temperatures are $1163^{\circ} \mathrm{C}, 1165^{\circ} \mathrm{C}$, $1166^{\circ} \mathrm{C}, 1168^{\circ} \mathrm{C}, 1170^{\circ} \mathrm{C}$ and $1171^{\circ} \mathrm{C}$. The batching plan with average holding temperature of $1163^{\circ} \mathrm{C}$ is listed in Table 4. 
Table 2. Batching planned by the traditional way.

\begin{tabular}{|c|c|c|c|c|c|c|c|c|c|c|c|c|}
\hline \multirow{2}{*}{ Workpiece Type } & \multicolumn{12}{|c|}{ Batch No. } \\
\hline & 1 & 2 & 3 & 4 & 5 & 6 & 7 & 8 & 9 & 10 & 11 & 12 \\
\hline $\mathrm{J} 1$ & & & 4 & & & & & & & & & \\
\hline $\mathrm{J} 2$ & & & & & 3 & & & & & & & \\
\hline $\mathrm{J} 3$ & & 12 & & & & & & & & & & \\
\hline $\mathrm{J} 4$ & & & & & & & 9 & & & & & \\
\hline $\mathrm{J} 5$ & & 11 & & & & & & & & & & \\
\hline J6 & & & & & & & 3 & & & & & \\
\hline J7 & & & & & & & & 2 & & & & \\
\hline $\mathrm{J} 8$ & & & & & & 6 & & & & & & \\
\hline J9 & & & & & & & & & & 13 & & \\
\hline $\mathrm{J} 10$ & 10 & & & & 6 & & & & & & & \\
\hline $\mathrm{J} 11$ & & & & & & & & & & & & 1 \\
\hline $\mathrm{J} 12$ & & & 6 & & & & & & & & & \\
\hline $\mathrm{J} 13$ & & & & & & & & & & & 5 & \\
\hline $\mathrm{J} 14$ & & & & 4 & & & & & & & & \\
\hline $\mathrm{J} 15$ & & & & 17 & & & & & & & & \\
\hline $\mathrm{J} 16$ & & & & & & & & & 7 & & & \\
\hline $\mathrm{J} 17$ & & & & & & & & 2 & & & & \\
\hline $\mathrm{J} 18$ & & & & & & 8 & & & & & & \\
\hline
\end{tabular}

Table 3. Parameters involved in algorithm.

\begin{tabular}{|c|c|}
\hline Parameter & Value \\
\hline \hline Population size r & 100 \\
\hline Number of iterations $\mathrm{n}$ & 0.8 \\
\hline Crossover probability p & 0.5 \\
\hline Mutation probability q & 0.6 \\
\hline Weight of quantity of batches $w_{k}$ & 0.3 \\
\hline Weight of average charging amount $w_{q}$ & 0.1 \\
\hline Weight of average holding temperature $w_{T}$ & 50 \\
\hline Number of repetitions $\mathrm{m}$ & 0.6 \\
\hline
\end{tabular}


Table 4. Optimal charging combination based on group genetic algorithm.

\begin{tabular}{|c|c|c|c|c|c|c|c|c|c|c|}
\hline Workpiece Type & 1 & 2 & 3 & 4 & 5 & 6 & 7 & 8 & 9 & 10 \\
\hline $\mathrm{J} 2$ & 2 & & & 1 & & & & & & \\
\hline $\mathrm{J} 4$ & 4 & 5 & & & & & & & & \\
\hline $\mathrm{J} 5$ & & & & & 11 & & & & & \\
\hline $\mathrm{J} 6$ & 2 & 1 & & & & & & & & \\
\hline $\mathrm{J} 10$ & & & 6 & 6 & & 4 & & & & \\
\hline J11 & & & & & & & & & & 1 \\
\hline $\mathrm{J} 12$ & 1 & & 2 & 1 & & 2 & & & & \\
\hline $\mathrm{J} 13$ & & & & & 5 & & & & & \\
\hline $\mathrm{J} 14$ & & & & & & & 4 & & & \\
\hline $\mathrm{J} 15$ & & & & & & & & 17 & & \\
\hline
\end{tabular}

\section{CONCLUSION}

The problem regarding how to separate work pieces with different holding temperature intervals and combine them for charging is described in this paper and a mathematical model of optimal charging combination for energy saving is proposed as well as a group genetic algorithm based on temperature compatibility rule proposed for problem solving. Additionally the above mentioned algorithm and the traditional batching planning are compared by an actual order for scheduling.

As seen from Table 2 and $\mathbf{4}$, the proposed algorithm described in this paper is better than the traditional one in terms of the number of batches, average charging amount and average holding temperature, resulting in less energy consumption. Therefore regarding the energy saving, the batching plan figured out by the proposed model and the algorithm described in this paper is of good functions.

Besides, for the charging batch with very small actual weight, it is recommended to arrange it into the next production planning cycle, or select some work pieces from the next production cycle to combine with it so as to form a new batch with weight close to maximum load capacity of furnace as much as possible (optimal charging amount). Also the lead time and the scheduling factors shall be considered in this case.

\section{CONFLICT OF INTEREST}

The authors confirm that this article content has no conflict of interest.

\section{ACKNOWLEDGEMENTS}

This work was financially supported by Project fund of Jiangsu Province Department of Education Philosophy and Social science (2012SJD630016). The supports are gratefully acknowledged. The authors would also acknowledge the Editor of International Journal of Advanced Manufacturing Technology.

\section{REFERENCES}

[1] Johansson M T, Söderström M. "Options for the Swedish steel industry-energy efficiency measures and fuel conversion" [J]. Energy, 2011, 36(1): 191-198. 
[2] Kasprzak W, Sokolowski J H, Yamagata H, et al. "Energy Efficient Heat Treatment for Linerless Hypereutectic Al-Si Engine Blocks Made Using Vacuum HPDC Process"[J]. Journal of Materials Engineering and Performance, 2011, 20(1): 120-132.

[3] Min Huang, Ruixian Huang, Bo Sun, Linrong Li. "Research on the Production Scheduling Optimization for Virtual Enterprises"[J]. Mathematical Problems in Engineering, vol. 2013, Article ID 492158, 9 pages, 2013. doi:10.1155/2013/492158.K. Elissa, "Title of paper if known," unpublished.

[4] FANG Y, ZHU B, LI D. "Research on Forging Production Plan and Control System Based on Fractal Holon"[J]. Machine Tool \& Hydraulics, 2011, 21: 3-5.

[5] Li Kai. "Research on Key-Machine Scheduling Problems with Consideration of Engery Conservation" [D].HeFei University of Technology,2009.

[6] Turgeon M, Giersch A, Delevoye-Turrell Y, et al. "Impaired predictive timing with spared time interval production in individual with schizophrenia"[J]. Psychiatry research, 2012, 197(1): 13-18.
[7] Poppe A, Halekas J S, Delory G T, et al. "Recent advances in understanding lunar surface charging: modeling, theory and spacecraft observations"[C]. AGU Fall Meeting Abstracts. 2012, 1: 03.

[8] Ghosh T, Sengupta S, Chattopadhyay M, et al. "Meta-heuristics in cellular manufacturing: A state-of-the-art review"[J]. International Journal of Industrial Engineering Computations, 2011, 2(1): 87122.

[9] Goncalves Filho V, José Tiberti A. "A group genetic algorithm for the machine cell formation problem"[J]. International Journal of Production Economics, 2006, 102(1): 1-21.

[10] Damodaran P, Rao A G, Mestry S. "Particle swarm optimization for scheduling batch processing machines in a permutation flowshop"[J]. The International Journal of Advanced Manufacturing Technology, 2013, 64(5-8): 989-1000.

[11] Zhang L, Chang H, Xu R. "Equal-width partitioning roulette wheel selection in genetic algorithm" $[\mathrm{C}]$. Technologies and Applications of Artificial Intelligence (TAAI), 2012 Conference on. IEEE, 2012: 62-67.

(C) Baiqing et al.; Licensee Bentham Open.

This is an open access article licensed under the terms of the (https://creativecommons.org/licenses/by/4.0/legalcode), which permits unrestricted, noncommercial use, distribution and reproduction in any medium, provided the work is properly cited. 